\section{Inactividad física versus sedentarismo: análisis de la Encuesta Nacional de Salud de Chile 2016-2017}

\author{
RODRIGO FERNÁNDEZ-VERDEJO ${ }^{1, a}$, MÓNICA SUÁREZ-REYES ${ }^{2, \text {, }}$
}

\section{Physical inactivity versus sedentariness: analysis of the chilean national health survey 2016-2017}

Background: Physical inactivity and sedentariness are independent risk factors for mortality. Physical inactivity is defined as engaging in insufficient moderate/vigorous physical activity (i.e. not meeting the WHO's recommendations). Sedentariness is defined according to sedentary behavior; evidence suggests that $>8 \mathrm{~h} / \mathrm{d}$ could serve to consider a person as sedentary. The Chilean National Health Survey 2016-2017 (NHS), using a single question (Question-NHS), considered as "sedentary" those who did not engage in sports or physical activity for $\geq 30 \mathrm{~min}, \geq 3$ times/wk. Thus, it attempted to estimate sedentariness without considering sedentary behavior. Aim: To determine the prevalence of physical inactivity and sedentariness in Chile, and to contrast such results with the Question-NHS. Material and Methods: We analyzed data from 5564 participants of the 2016-2017 NHS, aged $\geq 18$ years. The Global Physical Activity Questionnaire was used to determine moderate/vigorous physical activity and sedentary behavior. We defined physical inactivity as having $<600 \mathrm{MET} \times \mathrm{min} /$ wk of moderate/vigorous physical activity, and sedentariness as having $>8 \mathrm{~h} / \mathrm{d}$ of sedentary behavior. Results: The prevalences [95\% confidence intervals] of physical inactivity and sedentariness were 32\% [29-34] and 6\% [5-7] respectively, while 3\% [2-4] were both physically inactive and sedentary. The Question-NHS classified 88\% [86-89] as "sedentary", but among them, 35\% were physically inactive and 6\% were sedentary. Conclusions: One third of adults are inactive, one out of ten is sedentary, and one out of twenty is inactive and sedentary. The Question-NHS overestimates the population at risk.

(Rev Med Chile 2021; 149: 103-109)

Key words: Exercise; Public Health; Sedentary Behavior.
${ }^{1}$ Carrera de Nutrición y Dietética, Departamento de Ciencias de la Salud, Facultad de Medicina, Pontificia Universidad Católica de Chile. Santiago, Chile.

¿Escuela de Ciencias de la Actividad Física, el Deporte y la Salud (ECIADES), Universidad de Santiago de Chile. Santiago, Chile.

${ }^{\mathrm{a} P h D}$ en Ciencias de la

Motricidad

bPhD en Salud Pública.

Trabajo con Financiamiento Fondecyt de Iniciación \#11180361.

Los autores declaran no tener conflictos de interés.

Recibido el 9 de abril de 2019, aceptado el 6 de enero de 2021.

Correspondencia a:

Rodrigo Fernández-Verdejo Departamento de Ciencias de la Salud. Carrera de Nutrición y Dietética. Pontificia Universidad Católica de Chile.

Avenida Vicuña Mackenna 4686, Macul. Santiago, Chile.

rfernandev@uc.cl

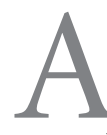

ctividad física se define como cualquier movimiento generado por los músculos esqueléticos que aumente el gasto energético por sobre el gasto de reposo ${ }^{1}$. Hay actividades de intensidades baja (<3,0 MET), moderada (3,0-5,9 MET) y vigorosa $(\geq 6 \mathrm{MET})^{1}$. Cuando la actividad es de muy baja intensidad ( $\leq 1,5 \mathrm{MET})$ se denomina comportamiento sedentario. Dependiendo de la actividad física a la que se refiera, su asociación con salud es beneficiosa o perjudicial.
Por un lado, mayores niveles de actividad física de intensidad moderada y/o vigorosa se asocian con menor mortalidad ${ }^{2-4}$. Es por ello que la OMS recomienda a los adultos acumular 150 $\mathrm{min} / \mathrm{sem}$ de actividad moderada, $75 \mathrm{~min} / \mathrm{sem}$ de actividad vigorosa, o una combinación de actividades moderadas y vigorosas que acumulen $\geq 600 \mathrm{MET} \times \mathrm{min} / \mathrm{sem}^{5,6}$. Por otro lado, mayores niveles de comportamiento sedentario se asocian con mayor mortalidad ${ }^{7,8}$. Pero no hay acuerdo res- 
pecto de la cantidad máxima de comportamiento sedentario que debe aconsejarse para reducir el riesgo para la salud ${ }^{9}$.

Las personas que no cumplen con las recomendaciones de actividad moderada y/o vigorosa se consideran como insuficientemente activas o inactivas $^{5,10}$. Ya que aún no existen recomendaciones globales respecto al comportamiento sedentario', no hay un punto de corte para clasificar a una persona como sedentaria. Siguiendo la lógica de la recomendación de actividad moderada y/o vigorosa, el punto de corte para clasificar a una persona como sedentaria debiese establecerse en función del tiempo de comportamiento sedentario que aumente la mortalidad. Un meta-análisis reciente mostró que la mortalidad aumenta por sobre $8 \mathrm{~h} / \mathrm{d}$ de comportamiento sedentario ${ }^{7}$; ese podría ser entonces un punto de corte adecuado. Importante, la inactividad física y el comportamiento sedentario son factores de riesgo independientes asociados a mortalidad ${ }^{8}$. De hecho, una persona puede ser al mismo tiempo físicamente activa (cumple las recomendaciones de actividad moderada y/o vigorosa) y sedentaria (acumula $>8 \mathrm{~h} / \mathrm{d}$ de comportamiento sedentario $)^{11}$. Es por ello esencial diferenciar inactividad física y sedentarismo para establecer los riesgos para la salud de la población.

La Encuesta Nacional de Salud de Chile 20162017 (ENS) reportó 86,7\% de "sedentarismo"12. Esa prevalencia se obtuvo a partir de una pregunta, que aquí llamaremos Pregunta-ENS. La Pregunta-ENS considera como "sedentarias" a las personas que: en los últimos 30 días no practicaron deporte o realizaron actividad física fuera de su horario de trabajo, durante 30 minutos o más cada vez, 3 o más veces por semana ${ }^{12}$. Los resultados de esa pregunta fueron también denominados "sedentarismo en el último mes" o "inactividad física"12,13. Esto presenta dos problemas fundamentales: (a) se quiso determinar sedentarismo sin considerar comportamiento sedentario, y (b) se usaron indistintamente los conceptos de inactividad física y sedentarismo. Estos problemas limitan la utilidad de este instrumento para informar sobre el riesgo para la salud en Chile.

Afortunadamente, la ENS además incluyó el Cuestionario Mundial de Actividad Física (GPAQ por sus siglas en ingles), que estima la actividad moderada y/o vigorosa, y el comportamiento sedentario. Este es el instrumento recomendado por la OMS para la vigilancia en relación con actividad física. En la sábana de datos de la ENS - publicada online-se reportó que 35,1\% de los mayores de 15 años tenía un "nivel bajo de actividad física según GPAQ". No está claro si eso equivale a la prevalencia de personas inactivas (i.e. $<600 \mathrm{MET} \times \mathrm{min} /$ sem); pero de ser así, las personas menores de 18 años debiesen haberse excluido del análisis, porque las recomendaciones de actividad física para ellas son distintas (i.e. $420 \mathrm{~min} / \mathrm{sem}$ de actividad moderada o vigorosa $)^{6}$.

Por lo tanto, actualmente se desconoce la prevalencia de inactividad física y sedentarismo (estimada por comportamiento sedentario) en personas adultas en Chile. Nuestro objetivo fue determinar esas prevalencias, y contrastar esos resultados con los de la Pregunta-ENS. Además, calculamos la prevalencia de las combinaciones de actividad física moderada y/o vigorosa y de sedentarismo.

\section{Material y Método}

Esta investigación empleó información de las Encuestas de Salud para vigilancia epidemiológica de la Subsecretaría de Salud Pública. Los autores agradecen al Ministerio de Salud de Chile haberle permitido disponer de la base de datos. Todos los resultados obtenidos del estudio son de responsabilidad de los autores y en nada comprometen a dicha institución. Se analizó la base de datos de la ENS 2016-2017, cuyo protocolo fue aprobado por el Comité Ético Científico de la Pontificia Universidad Católica de Chile.

\section{Actividad física moderada y/o vigorosa}

Analizamos el GPAQ aplicado en la ENS. El GPAQ incluye preguntas sobre actividad física de intensidad moderada y vigorosa en el trabajo, transporte y tiempo libre. Registra el tiempo dedicado a cada actividad y su frecuencia en una semana típica. Asume un requerimiento energético de 4 y 8 MET para actividades moderadas y vigorosas, respectivamente ${ }^{5}$. La actividad física de intensidad moderada y/o vigorosa se expresa en MET $\times$ min/ sem. Inactividad física se definió como acumular $<600 \mathrm{MET} \times \mathrm{min} / \mathrm{sem}$ de actividad moderada $\mathrm{y} / \mathrm{o}$ vigorosa, según las recomendaciones ${ }^{5,6}$. Nótese que ese punto de corte equivale a acumular 150 $\mathrm{min} / \mathrm{sem}$ de actividad moderada $(150 \mathrm{~min} / \mathrm{sem}$ $\times 4 \mathrm{MET}), 75 \mathrm{~min} / \mathrm{sem}$ de actividad vigorosa (75 $\mathrm{min} / \mathrm{sem} \times 8 \mathrm{MET}$ ), o una combinación de ambos. 


\section{Sedentarismo}

El GPAQ también estima comportamiento sedentario al registrar el tiempo sentado y/o recostado (excluyendo el tiempo durmiendo) ${ }^{5}$. Definimos sedentarismo como acumular $>8 \mathrm{~h} / \mathrm{d}$ de comportamiento sedentario, según el metaanálisis de Patterson et al. ${ }^{7}$ que mostró mayor mortalidad a partir de esos niveles. También calculamos el "sedentarismo" según la Pregunta-ENS, que considera como "sedentarias" a las personas que en los últimos 30 días no practicaron deporte o realizaron actividad fisica fuera de su horario de trabajo, durante 30 minutos o más cada vez, 3 o más veces por semana ${ }^{12}$.

\section{Grupos según actividad física moderada $y / o$ vigorosa y sedentarismo}

Usando los resultados del GPAQ, las personas fueron clasificadas como activas o inactivas, y como sedentarias o no-sedentarias (según el criterio de Patterson et al. ${ }^{7}$ ). Cuatro grupos de personas fueron así identificados: activas/no-sedentarias, activas/sedentarias, inactivas/no-sedentarias, e inactivas/sedentarias. La prevalencia de estos grupos fue calculada para identificar grupos de riesgo en función del patrón de actividad física: dos factores de riesgo (inactividad y sedentarismo), un factor de riesgo (inactividad o sedentarismo), sin factor de riesgo (activas/no-sedentarias).

\section{Análisis}

La muestra total de la ENS incluyó 6.233 personas. Sin embargo, en el presente reporte sólo consideramos las personas $\geq 18$ años que respondieron el GPAQ correctamente. Específicamente, excluimos las personas que: (a) tuvieron respuestas inconsistentes, ej. responder que no realiza actividad vigorosa en el trabajo, pero luego reportar una cantidad de tiempo; (b) reportaron $<10 \mathrm{~min}$ en un determinado subdominio (ej. actividad vigorosa en el trabajo), porque el GPAQ sólo considera actividades en períodos de $\geq 10 \mathrm{~min}$ consecutivos $^{5}$; (c) reportaron $>16 \mathrm{~h} / \mathrm{d}$ de actividad en un subdominio (ej. actividad vigorosa en el trabajo $)^{5}$; $\mathrm{y}$ (d) reportaron $>10.080 \mathrm{~min} / \mathrm{sem}$ al considerar su actividad moderada y/o vigorosa más su comportamiento sedentario. También excluimos outliers extremos para la actividad moderada y/o vigorosa y para el comportamiento sedentario, usando las fórmulas ${ }^{13,14}$ : Límite inferior $=\mathrm{Q} 1-3 \times$ [Q3-Q1]; Límite superior = Q3 + 3× [Q3-Q1].

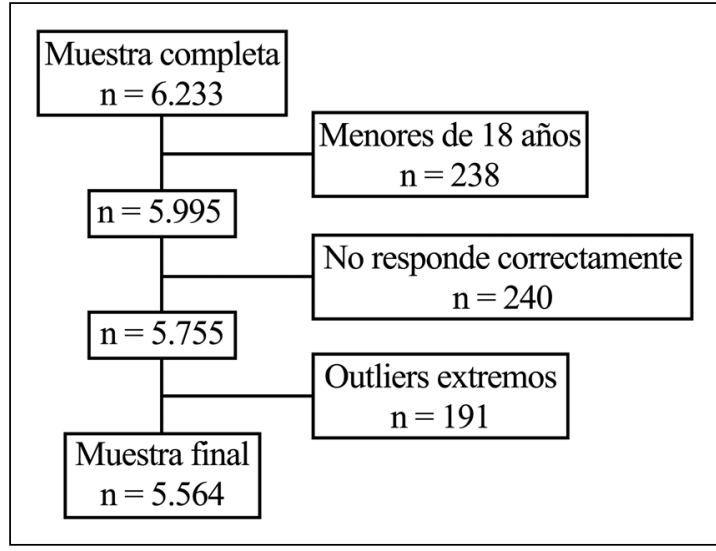

Figura 1. Diagrama de flujo para la selección de la muestra.

En total, 5.564 personas cumplieron estos criterios de inclusión, cuyos resultados se presentan en este reporte. La Figura 1 muestra el diagrama de flujo para la selección de la muestra.

En los análisis se consideró el diseño muestral complejo de la ENS, y se aplicaron los factores de expansión adecuados. Para evitar romper la estructura de la ENS por la exclusión de participantes, los análisis fueron no condicionados e incluyeron todos los participantes, considerando como una subpoblación a aquellos que cumplieron los criterios de inclusión ${ }^{15}$. Como análisis de sensibilidad, repetimos los análisis principales sin excluir outliers extremos. Se usó el módulo de muestras complejas del programa IBM $^{\circledR}$ SPSS $^{\circledR}$ Statistics v.26 para todos los análisis.

\section{Resultados}

\section{Prevalencia de inactividad física y sedentarismo}

La Tabla 1 muestra el promedio [IC95\%] de edad, actividad física moderada y/o vigorosa, $y$ comportamiento sedentario para el total de participantes y estratificados por sexo. Considerando el total de participantes, la prevalencia [IC95\%] de inactividad física fue 31,5\% [29,2-33,9], siendo mayor en mujeres que en hombres (Tabla 1 ). La prevalencia de sedentarismo según el criterio de Patterson et al. ${ }^{7}$ fue 5,8\% [4,8-7,2] en el total de participantes, sin diferencias entre sexos (Tabla 1). En contraste, la prevalencia de "sedentarismo" según la Pregunta-ENS alcanzó 87,6\% [85,789,2 ], siendo mayor en mujeres que en hombres (Tabla 1). 
Tabla 1. Inactividad física y sedentarismo en personas adultas en Chile

\begin{tabular}{|c|c|c|c|}
\hline Variables & Total & Mujeres & Hombres \\
\hline \multicolumn{4}{|l|}{ Muestra expandida } \\
\hline$n$ & $\begin{array}{c}12.631 .400 \\
{[11.826 .762-13.436 .038]}\end{array}$ & $\begin{array}{c}6.554 .567 \\
{[6.062 .882-7.046 .253]}\end{array}$ & $\begin{array}{c}6.076 .832 \\
{[5.576 .742-6.576 .923]}\end{array}$ \\
\hline$\%$ & 100 & $51,9[49,6-54,2]$ & $48,1[45,8-50,4]$ \\
\hline Edad (años) & $44,9[44,0-45,8]$ & $45,7[44,6-46,7]$ & $44,1[42,7-45,5]$ \\
\hline $\begin{array}{l}\text { Actividad física de intensidad moderada } \\
\text { y/o vigorosa }(\mathrm{MET} \times \mathrm{min} / \mathrm{sem})\end{array}$ & $4.925[4.548-5.302]$ & $3.466[3.133-3.799]$ & $6.498[5.885-7.110]$ \\
\hline Comportamiento sedentario $(\mathrm{h} / \mathrm{d})$ & $3,1[3,0-3,3]$ & $2,9[2,7-3,1]$ & $3,4[3,1-3,6]$ \\
\hline \multicolumn{4}{|c|}{ Actividad física de intensidad moderada y/o vigorosa } \\
\hline Inactiva, < $600 \mathrm{MET} \times \mathrm{min} / \mathrm{sem}(\%)$ & $31,5[29,2-33,9]$ & $38,8[35,4-42,4]$ & $23,6[20,7-26,7]$ \\
\hline Activa, $\geq 600 \mathrm{MET} \times \mathrm{min} / \mathrm{sem}(\%)$ & $68,5[66,1-70,8]$ & $61,2[57,6-64,6]$ & $76,4[73,3-79,3]$ \\
\hline \multicolumn{4}{|c|}{ Sedentarismo según criterio de Patterson et al. 2018} \\
\hline Sí, > $8 \mathrm{~h} / \mathrm{d}$ de comportamiento sedentario (\%) & $5,8[4,8-7,2]$ & $4,8[3,6-6,4]$ & $7,0[5,3-9,2]$ \\
\hline No, $\leq 8 \mathrm{~h} / \mathrm{d}$ de comportamiento sedentario (\%) & $94,2[92,8-95,2]$ & $95,2[93,6-96,4]$ & $93,0[90,8-94,7]$ \\
\hline \multicolumn{4}{|l|}{ Sedentarismo según Pregunta-ENS } \\
\hline $\begin{array}{r}\text { Si, }<3 \text { veces/sem de deporte/actividad } \\
\text { física fuera del trabajo (\%) }\end{array}$ & $87,6[85,7-89,2]$ & $90,3[88,2-92,0]$ & $84,7[81,6-87,4]$ \\
\hline $\begin{array}{r}\text { No, } \geq 3 \text { veces/sem de deporte/actividad } \\
\text { física fuera del trabajo (\%) }\end{array}$ & $12,4[10,8-14,3]$ & $9,7[8,0-11,8]$ & $15,3[12,6-18,4]$ \\
\hline
\end{tabular}

Los datos representan promedio o porcentaje [intervalo de confianza al 95\%].

Similares resultados se obtuvieron sin excluir los outliers extremos (análisis de sensibilidad; $\mathrm{n}=5.755)$. Considerando el total de participantes, la prevalencia de inactividad física fue $30,9 \%$ [28,7-33,2], y de sedentarismo según el criterio de Patterson et al. ${ }^{7}$ fue 6,5\% [5,4-7,8]. En contraste, la prevalencia de "sedentarismo" según la Pregunta-ENS fue 87,7\% [85,9-89,3].

\section{Comparación entre resultados del GPAQ y Pregunta-ENS}

La prevalencia de "sedentarismo" según la Pregunta-ENS fue notablemente mayor a la prevalencia de inactividad física y de sedentarismo (según Patterson et al. ${ }^{7}$ ) calculadas con el GPAQ. De hecho, entre aquellas personas clasificadas como "sedentarias" según la Pregunta-ENS, sólo $34,9 \%$ [32,4-37,5] clasificó como inactiva (Figura 2A), y sólo 5,9\% [4,8-7,2] clasificó como sedentaria según el criterio de Patterson et al. ${ }^{7}$ usando el GPAQ (Figura 2B). Similares tendencias se observaron en los análisis estratificados por sexo (Mujeres: inactivas 41,6\% [37,9-45,4], sedentarias 4,6\% [3,4-6,2] según Patterson et al. 7 ; Hombres: inactivos 27,2\% [23,9-30,7], sedentarios 7,4\% $[5,6-9,7]$ según Patterson et al. ; Figura 2).

\section{Prevalencia de grupos de actividad física moderada y/o vigorosa $y$ de sedentarismo}

La Figura 3 muestra la prevalencia de personas inactivas/sedentarias, inactivas/no-sedentarias, activas/sedentarias, y activas/no-sedentarias en el total de participantes y estratificados por sexo. Nótese que 34\% del total de participantes tiene al menos un factor de riesgo asociado al patrón de actividad física (inactividad o sedentarismo), y 3,3\% tiene ambos (inactividad y sedentarismo). Los respectivos valores en mujeres son $40,7 \%$ y $2,9 \%$, mientras que en hombres son $26,8 \%$ y $3,7 \%$. 
A

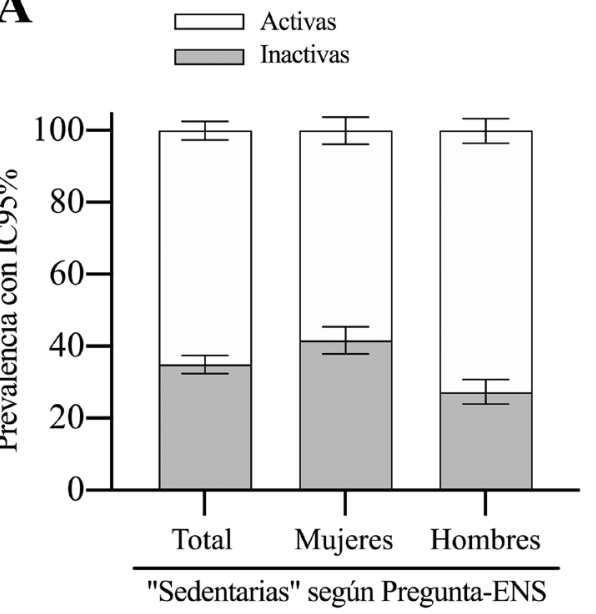

B

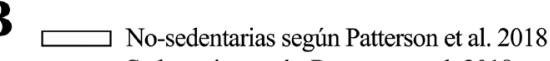

Sedentarias según Patterson et al. 2018

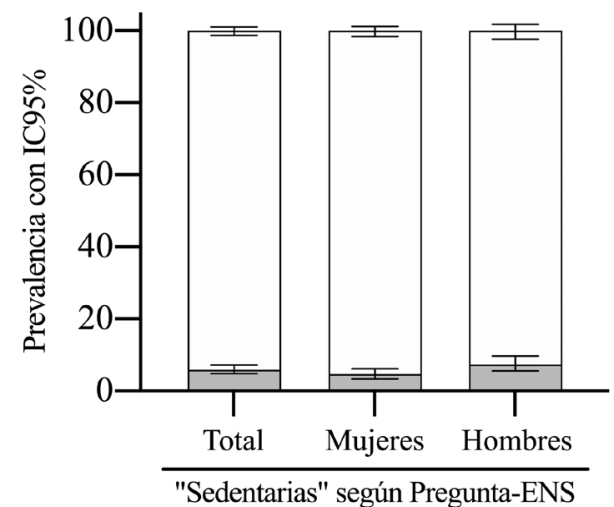

Figura 2. Prevalencia de inactividad física y sedentarismo (según criterio de Patterson et al. 2018) en las personas clasificadas como "sedentarias" según la Pregunta-ENS. (A) Prevalencia de personas físicamente activas ( $\geq 600 \mathrm{MET} \times \mathrm{min} / \mathrm{sem}$ de actividad moderada y/o vigorosa) e inactivas (<600 MET x min/sem de actividad moderada y/o vigorosa). (B) Prevalencia de personas sedentarias ( $>8 \mathrm{~h} / \mathrm{d}$ de comportamiento sedentario) y no-sedentarias ( $\leq 8 \mathrm{~h} / \mathrm{d}$ de comportamiento sedentario).

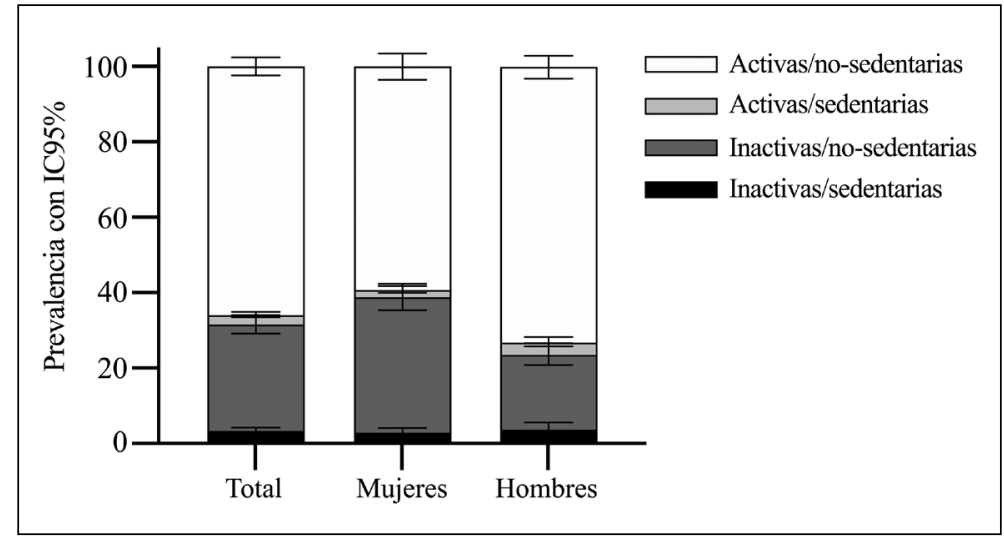

Figura 3. Prevalencia de grupos de personas según actividad física moderada y/o vigorosa y sedentarismo. Activas, $\geq 600 \mathrm{MET} \times \mathrm{min} / \mathrm{sem}$ de actividad moderada y/o vigorosa; Inactivas, $<600$ MET $\times$ min/sem de actividad moderada y/o vigorosa; Sedentarias, $>8 \mathrm{~h} / \mathrm{d}$ de comportamiento sedentario; No-sedentarias, $\leq 8 \mathrm{~h} / \mathrm{d}$ de comportamiento sedentario.

\section{Discusión}

Nuestros resultados muestran que la prevalencia de inactividad física en personas adultas en Chile es 31,5\%. La prevalencia de sedentarismo, según el criterio de Patterson et al. ${ }^{7}$, es 5,8\%. Ambas prevalencias difieren radicalmente de la prevalencia de "sedentarismo" según la Pregunta-ENS, que en nuestros cálculos alcanzó 87,6\% (similar al $86,7 \%$ previamente reportado al considerar toda la población ${ }^{12}$ ). Nuestros datos -basados en estándares internacionales- evidencian que la mayoría de las personas "sedentarias" según la Pregunta-ENS son físicamente activas, y sólo un bajo porcentaje son sedentarias. Estas tendencias fueron encontradas en ambos sexos. De modo que la Pregunta-ENS sobreestima la población en riesgo.

Un estudio previo estimó la prevalencia mundial de inactividad física en $27,5 \%{ }^{10}$. En países latinoamericanos y del caribe dicha prevalencia alcanzó 39,1\%. Ese estudio utilizó cuestionarios con preguntas sobre actividad en el trabajo, transporte, y tiempo libre. El GPAQ fue uno de ellos. 
Nuestros datos a partir del GPAQ indican que la inactividad física en Chile sigue las prevalencias mundiales. Un tercio de la población no cumple las recomendaciones de actividad moderada y/o vigorosa, y esa prevalencia es mayor en mujeres que en hombres ${ }^{10}$. Importante, la prevalencia de inactividad física en Chile es mayor a la prevalencia de otros indicadores, ej. consumo riesgoso de alcohol $(12,7 \%)$ y diabetes mellitus $(12,7 \%)^{13}$. Por lo que la inactividad física sigue siendo uno de los factores de riesgo más prevalentes en Chile.

Para sedentarismo no hay una definición establecida. Como una primera aproximación, aquí consideramos sedentarias las personas con $>8 \mathrm{~h} / \mathrm{d}$ de comportamiento sedentario, ya que esos niveles se han asociado con mayor mortalidad ${ }^{7}$. Encontramos una prevalencia de sedentarismo de sólo $5,8 \%$, pero se requieren estudios adicionales para establecer si el punto de corte utilizado es el más adecuado.

En cambio, utilizando la Pregunta-ENS la prevalencia de "sedentarismo" fue 87,6\%. Creemos que este instrumento es inadecuado para determinar sedentarismo y para determinar inactividad física, porque: (a) pregunta sobre actividad física o deporte, pero actividad física -conceptualmenteincluye el comportamiento sedentario, mientras que deportes son actividades que pueden ser desde sedentarias (Tai Chi: 1,5 MET) hasta vigorosas (baloncesto: 8,0 MET) ${ }^{16}$; (b) intenta determinar sedentarismo sin preguntar por comportamiento sedentario; (c) se desconoce su asociación con salud; y (d) sobreestima la prevalencia de inactividad física y de sedentarismo. Los resultados de esta pregunta no son entonces un buen indicador del riesgo para la salud. Además, sus limitaciones conceptuales confunden a las comunidades médica y académica, y también a la población general.

La inactividad física y el comportamiento sedentario son factores de riesgo de mortalidad independientes asociados al patrón de actividad ${ }^{8}$. Aquí calculamos la prevalencia de los diferentes grupos de actividad moderada y/o vigorosa y sedentarismo que pueden identificarse. Encontramos que un tercio de las personas adultas en Chile tiene al menos uno de los factores de riesgo (inactividad o sedentarismo), mientras que menos del $5 \%$ presenta ambos. Previamente se ha reportado que-comparadas con personas activas/no-sedentarias-las personas activas/sedentarias, inactivas/ no-sedentarias, e inactivas/sedentarias tienen ma- yor prevalencia de síndrome metabólico ${ }^{11}$. Importante, las personas inactivas/sedentarias -ademáspresentaron mayor prevalencia de enfermedades crónicas, obesidad, obesidad abdominal, presión arterial elevada, glicemia elevada, trigliceridemia elevada, y HDL-C disminuido ${ }^{11}$. Estos resultados demuestran como la combinación de los factores de riesgo asociados al patrón de actividad perjudican la salud. Discriminar ambos factores puede ser entonces relevante para determinar el riesgo en la salud de la población.

Los cuestionarios de actividad física -como el GPAQ- han permitido entender la asociación entre actividad física y mortalidad. No obstante, estos cuestionarios se enfocan sólo en actividades de intensidad moderada y/o vigorosa realizadas en $\geq 10$ min consecutivos ${ }^{5}$. Usando mediciones objetivas de actividad física con acelerómetros, estudios recientes han demostrado que mayores niveles de actividad de baja intensidad $(>1,5$ hasta $<3,0$ MET) también se asocian con menor mortalidad $^{8}$. También se ha demostrado que no es necesario acumular las actividades en períodos de $\geq 10$ min consecutivos ${ }^{8,17}$. La medición objetiva de la actividad física en todo su rango de intensidades (desde comportamiento sedentario hasta actividades vigorosas) permitirá determinar de manera más precisa el riesgo para la salud de la población. En Chile, debiésemos aspirar a este tipo de mediciones en el futuro, como ya se hace en otros países ${ }^{11,18}$.

En futuras encuestas, se debiese informar sobre inactividad física usando los resultados del GPAQ y las recomendaciones internacionales. Así se podrán comparar los resultados de Chile con otras poblaciones. También se podrá reconocer mejor el riesgo para la salud asociado a la inactividad física en nuestro país.

Agradecimientos: Al Ministerio de Salud de Chile por proporcionar la base de datos. A José Galgani, Alice Glaves, Lorena Malo-Vintimilla, y Eduardo Fuentes-López (Pontificia Universidad Católica de Chile) por su retroalimentación.

\section{Referencias}

1. Garber CE, Blissmer B, Deschenes MR, Franklin BA, Lamonte MJ, Lee IM, et al. Quantity and quality of exercise for developing and maintaining cardiorespiratory, 
musculoskeletal, and neuromotor fitness in apparently healthy adults: Guidance for prescribing exercise. Med Sci Sports Exerc 2011; 43 (7): 1334-59.

2. Arem H, Moore SC, Patel A, Hartge P, Berrington de González A, Visvanathan K, et al. Leisure time physical activity and mortality: a detailed pooled analysis of the dose-response relationship. JAMA Intern Med [Internet] 2015; 175 (6): 959-67. Available from: http://www. ncbi.nlm.nih.gov/pubmed/25844730.

3. Wen CP, Wai JPM, Tsai MK, Yang YC, Cheng TYD, Lee M-C, et al. Minimum amount of physical activity for reduced mortality and extended life expectancy: a prospective cohort study. Lancet (London, England) [Internet] 2011; 378 (9798): 1244-53. Available from: http://www.ncbi.nlm.nih.gov/pubmed/21846575.

4. Lear SA, Hu W, Rangarajan S, Gasevic D, Leong D, Iqbal $\mathrm{R}$, et al. The effect of physical activity on mortality and cardiovascular disease in 130000 people from 17 high-income, middle-income, and low-income countries: the PURE study. Lancet (London, England) [Internet] 2017; 390 (10113): 2643-54. Available from: http://www. ncbi.nlm.nih.gov/pubmed/28943267.

5. World Health Organization. Global Physical Activity Questionnaire (QPAQ). Analysis Guide [Internet]. Available from: https://www.who.int/ncds/surveillance/ steps/GPAQ_EN.pdf.

6. Organización Mundial de la Salud. Recomendaciones mundiales sobre actividad física para la salud. Geneva; 2010.

7. Patterson R, McNamara E, Tainio M, de Sá TH, Smith $\mathrm{AD}$, Sharp SJ, et al. Sedentary behaviour and risk of all-cause, cardiovascular and cancer mortality, and incident type 2 diabetes: a systematic review and dose response meta-analysis. Eur J Epidemiol [Internet] 2018; 33 (9): 811-29. Available from: http://www.ncbi.nlm. nih.gov/pubmed/29589226.

8. Ekelund U, Tarp J, Steene-Johannessen J, Hansen BH, Jefferis B, Fagerland MW, et al. Dose-response associations between accelerometry measured physical activity and sedentary time and all cause mortality: systematic review and harmonised meta-analysis. BMJ [Internet]. 2019;366:14570. Available from: http://www.ncbi.nlm. nih.gov/pubmed/31434697.

9. Chaput J-P, Olds T, Tremblay MS. Public health guidelines on sedentary behaviour are important and needed: a provisional benchmark is better than no benchmark at all. Br J Sports Med [Internet] 2020; 54 (5): 308-9. Available from: http://www.ncbi.nlm.nih.gov/pub$\mathrm{med} / 30413423$.

10. Guthold R, Stevens GA, Riley LM, Bull FC. Worldwide trends in insufficient physical activity from 2001 to 2016: a pooled analysis of 358 population-based surveys with 1.9 million participants. Lancet Glob Heal [Internet] 2018; 6 (10): e1077-86. Available from: http:// dx.doi.org/10.1016/S2214-109X(18)30357-7.

11. Thakkar N, Jamnik V, Ardern CI. Cross-associations between physical activity and sedentary time on metabolic health: a comparative assessment using self-reported and objectively measured activity. J Public Health (Oxf) [Internet] 2018; 40 (4): e464-73. Available from: http:// www.ncbi.nlm.nih.gov/pubmed/29659929.

12. Departamento de Epidemiología Ministerio de Salud de Chile. Documento presentación primeros resultados. Tercera Encuesta Nacional de Salud (ENS) 2016-2017. 2017.

13. Departamento de Epidemiología Ministerio de Salud de Chile. Encuesta Nacional de Salud 2016-2017. Informe Final. Ministerio de Salud, Gobierno de Chile. 2017.

14. Fernández-Verdejo R, Moya-Osorio JL, Fuentes-López E, Galgani JE. Metabolic health and its association with lifestyle habits according to nutritional status in Chile: A cross-sectional study from the National Health Survey 2016-2017. Tauler P, editor. PLoS One [Internet] 2020; 15 (7): e0236451. Available from: https://dx.plos. org/10.1371/journal.pone.0236451.

15. West BT, Berglund P, Heeringa SG. A closer examination of subpopulation analysis of complex-sample survey data. Stata J 2008; 8 (4): 520-31.

16. Ainsworth BE, Haskell WL, Herrmann SD, Meckes N, Bassett DR, Tudor-Locke C, et al. 2011 compendium of physical activities: A second update of codes and MET values. Med Sci Sports Exerc 2011; 43 (8): 1575-81.

17. Saint-Maurice PF, Troiano RP, Matthews CE, Kraus WE. Moderate-to-Vigorous Physical Activity and All-Cause Mortality: Do Bouts Matter? J Am Heart Assoc [Internet] 2018; 7 (6). Available from: http://www. ncbi.nlm.nih.gov/pubmed/29567764.

18. Troiano RP, Berrigan D, Dodd KW, Mâsse LC, Tilert T, McDowell M. Physical activity in the United States measured by accelerometer. Med Sci Sports Exerc [Internet] 2008; 40 (1): 181-8. Available from: http://www. ncbi.nlm.nih.gov/pubmed/18091006. 